\title{
Experimental Investigation of Tape Springs Folded in Three Dimensions
}

\author{
Scott J. I. Walker* and Guglielmo S. Aglietti ${ }^{\dagger}$ \\ University of Southampton, Southampton, England SO17 1BJ, United Kingdom
}

\begin{abstract}
One of the most significant drivers in satellite design is the minimization of mass to reduce the large costs involved in the launch. With technological advances across many fields, it is now widely known that very low-mass satellites can perform a wide variety of missions. However, there is a need for small, efficient, area deployment devices. One possible structural solution for such devices is tape springs. Previous work on tape spring hinges has focused on two-dimensional folds; however, applications exist that incorporate three-dimensional tape spring folds. The properties of three-dimensional tape spring folds are experimentally investigated using a specially designed test rig. The rig is first used to produce comparative two-dimensional data before being used to analyze more complex three-dimensional folds.
\end{abstract}

\begin{tabular}{|c|c|}
\hline & Nomenclature \\
\hline$L$ & $=$ total tape spring length \\
\hline$M$ & $\begin{aligned} &= \text { bending moment applied to the } \\
& \text { strip (about } y \text { axis) }\end{aligned}$ \\
\hline$M_{H}$ & $\begin{aligned}= & \text { moment about array hinge line } \\
& (\text { skewed system) }, \mathrm{N} \cdot \mathrm{mm}\end{aligned}$ \\
\hline$M_{H}^{\max }$ & $\begin{array}{l}=\begin{array}{l}\text { peak hinge moment in the } \\
\text { skewed system, } \mathrm{N} \cdot \mathrm{mm}\end{array}\end{array}$ \\
\hline$M_{H+}^{\max }, M_{H-}^{\max }$ & $\begin{array}{l}=\text { peak buckling moments for two-dimensional } \\
\text { opposite and equal sense bends, respectively, } \\
\text { skewed system, } \mathrm{N} \cdot \mathrm{mm}\end{array}$ \\
\hline$M_{H}^{*}$ & $\begin{array}{l}=\quad \text { steady-state hinge moment in the } \\
\text { skewed system, } \mathrm{N} \cdot \mathrm{mm}\end{array}$ \\
\hline$M_{H+}^{*}, M_{H-}^{*}$ & $\begin{aligned}= & \text { steady-state moments for two-dimensional } \\
& \text { opposite and equal sense bends, respectively, } \\
& \text { skewed system, } \mathrm{N} \cdot \mathrm{mm}\end{aligned}$ \\
\hline$M^{\max }$ & $=$ peak moment in the theoretical system \\
\hline$M_{+}^{\max }, M_{-}^{\max }$ & $\begin{array}{l}=\text { peak buckling moments for two-dimensional } \\
\text { opposite and equal sense bends, respectively, } \\
\text { theoretical system, } \mathrm{N} \cdot \mathrm{mm}\end{array}$ \\
\hline$M_{+}^{*}, M_{-}^{*}$ & $\begin{aligned} &= \text { steady-state moments for two-dimensional } \\
& \text { opposite and equal sense bends, respectively, } \\
& \text { theoretical system, } \mathrm{N} \cdot \mathrm{mm}\end{aligned}$ \\
\hline$M^{*}$ & $=$ steady-state moment in the theoretical system \\
\hline$R_{y}$ & $\begin{aligned}= & \text { initial transverse radius of curvature } \\
& (\text { along } y), \mathrm{mm}\end{aligned}$ \\
\hline$T$ & twisting torque applied to tape, $\mathrm{N} \cdot \mathrm{mm}$ \\
\hline$T_{+}, T_{-}$ & $\begin{aligned}= & \text { opposite and equal sense bend torques } \\
& \text { respectively, } \mathrm{N} \cdot \mathrm{mm}\end{aligned}$ \\
\hline$t$ & $=$ tape spring thickness, $\mathrm{mm}$ \\
\hline$x, y, z$ & $=$ stationary coordinate system \\
\hline$x^{\prime}, y^{\prime}, z^{\prime}$ & $\begin{array}{l}=\text { moving coordinate system (fixed relative to the } \\
\text { tape end) }\end{array}$ \\
\hline$\alpha$ & $\begin{array}{l}=\text { tape spring cross-sectional } \\
\text { angle of embrace, rad }\end{array}$ \\
\hline$\gamma$ & $=$ relative twist angle between tape ends \\
\hline
\end{tabular}

Received 11 January 2005; revision received 1 June 2005; accepted for publication 13 July 2005. Copyright (C) 2005 by Scott J. I. Walker and Guglielmo S. Aglietti. Published by the American Institute of Aeronautics and Astronautics, Inc., with permission. Copies of this paper may be made for personal or internal use, on condition that the copier pay the $\$ 10.00$ per-copy fee to the Copyright Clearance Center, Inc., 222 Rosewood Drive, Danvers, MA 01923; include the code 0001-1452/06 \$10.00 in correspondence with the CCC.

*Research Fellow, Astronautics Research Group, School of Engineering Sciences.

${ }^{\dagger}$ Senior Lecturer in Aerospace Structural Dynamics, School of Engineering Sciences. Member AIAA.

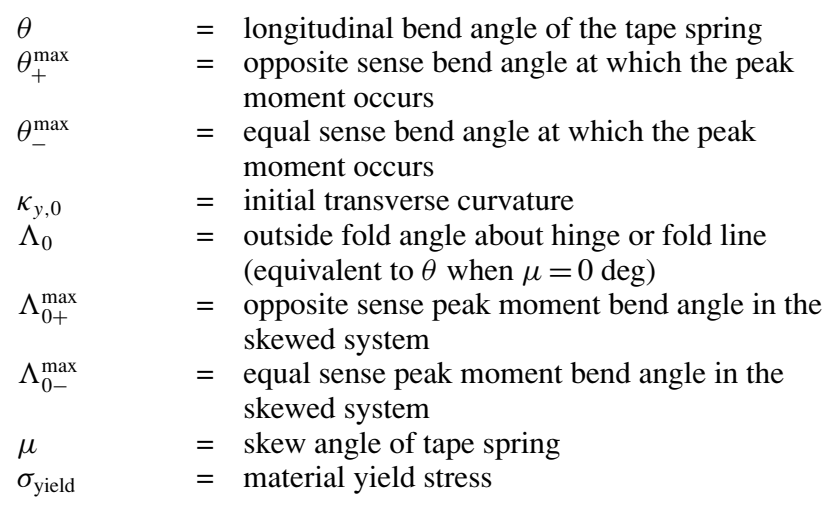

\section{Introduction}

O VER the past two decades there has been growing research and development into small satellite technologies due to the inherant cost savings of a reduction in the satellite mass. However, as the total satellite mass reduces, the power requirement of the satellite, in many applications, does not reduce at the same rate. For example, the small size of cellular telephones and hand-held receivers for the global positioning satellite navigation system creates a need for larger antenna sizes in the space-based components of the system. Greater capacity requires greater onboard power, provided by deployed solar arrays. There is also ongoing research into the use of ion propulsion systems, as primary and secondary propulsion for small satellites, due to their low mass potential. ${ }^{1}$ Such systems would also significantly increase the power requirement of the satellite. Usually micro- and nanosatellites employ body-mounted solar cells. However, there is growing interest in deploying photovoltaic cell areas larger than the surface area of the satellite. Small, cheap, reliable, and efficient deployment methods are, therefore, required to exploit the full potential of future small satellite missions. One of the main structural components that meets the low-mass requirement due to its efficiency is a tape spring. A tape spring is a metal tape with a curved cross section like a carpenter tape.

All previous studies involving tape springs have incorporated tape springs either folded in two dimensions (where the central line of the fold is perpendicular to the lengthwise direction) or rolled up (such as carpenter tape). The objective of this work is to experimentally investigate the properties of three-dimensional tape spring folds, that is, where the central line of the fold does not remain perpendicular to the lengthwise direction (Fig. 1). Such folds can be used to create efficient small satellite area deployment devices. In this work the term fold line refers to this central line of the fold about which the ends are rotated. For the two-dimensional case it is equivalent to a line of principle curvature of the surface. ${ }^{2}$ 


\section{Background}

Tape springs are defined as thin metallic strips with a curved cross section, which have the key property that they are continuous, that is, contain no mechanical hinges or other folding devices, and yet they can still be folded elastically. The general coordinate system used throughout this study is shown in Fig. 2. The $x$ axis is defined as running along the longitudinal length of the tape spring, the $y$ axis lies horizontally in the sectional plane of the tape spring, and the $z$ axis is normal to the longitudinal centerline of the tape. The origin is located at the centroid of the section such that the end rotations occur about the $y$ axis.

The standard tape spring geometry for theoretical analysis is defined by four initial parameters (three in the sectional plane and one defining the length). These can be seen in Fig. 3.

When a tape spring is subjected to two equal and opposite applied moments $M$ (acting about the $y$ direction), the tape spring initially deflects, then buckles to form a localized fold as shown in Fig. 4.

Depending on the direction of the applied moments, the tape spring will buckle, producing a longitudinal radius of curvature either on the same side, or the opposing side to the initial transverse radius of curvature. These folds are known as two-dimensional equal and opposite sense bends, respectively. The general relationship between $M$ and $\theta$ can be seen in Fig. 5, where opposite and equal sense buckling occurs at points A and B, respectively.

The general sign convention for this relationship is that a positive moment $M$ produces an opposite sense bend ${ }^{3}$ as shown in Fig. 4. From Fig. 5, note that four key moment values are identified for

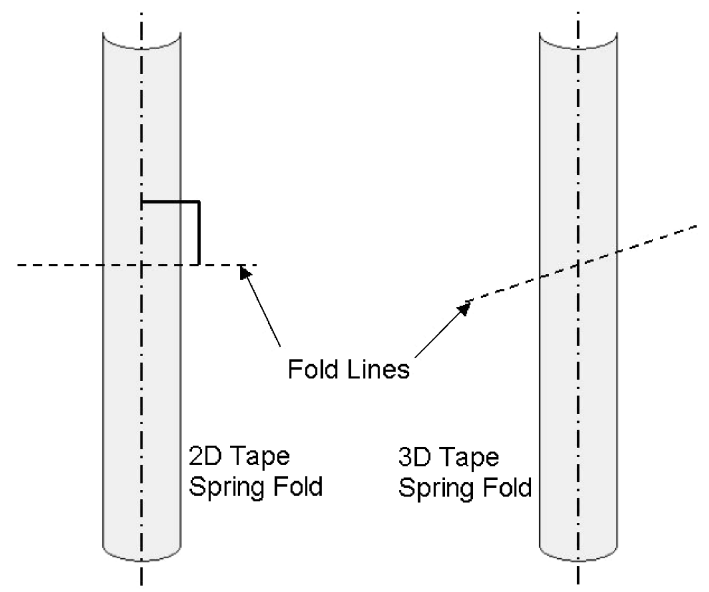

Fig. 1 Fold lines overlaid on tape spring.

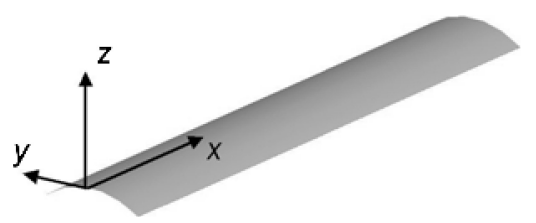

Fig. 2 General tape spring coordinate system.
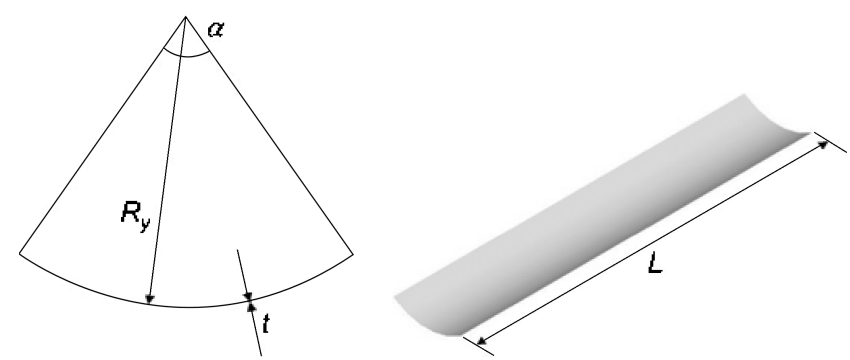

a)

b)

Fig. 3 General tape spring parameters.
Fig. 4 Tape spring rotated by $\theta$ in an opposite sense bend.
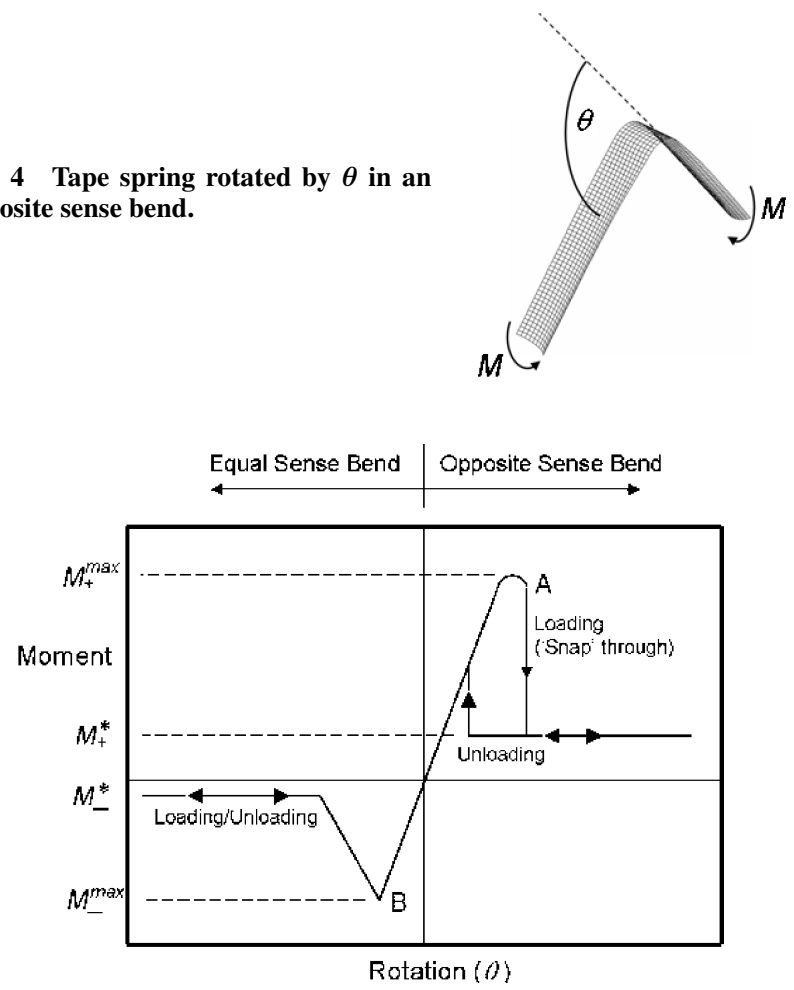

Fig. 5 Moment-rotation relationship for two-dimensional tape spring fold.
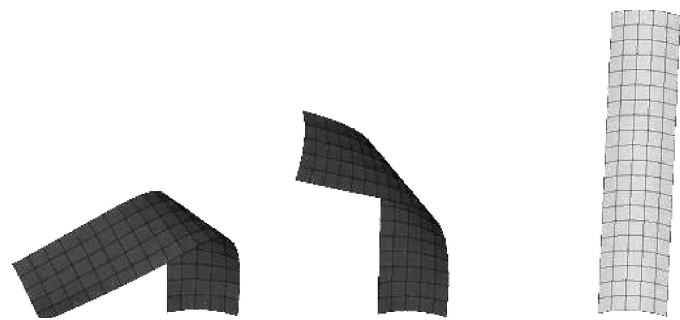

Fig. 6 Example of three-dimensional tape spring fold.

two-dimensional bends. These are the peak buckling moments $M_{+}^{\max }$ and $M_{-}^{\max }$, for opposite and equal sense bends, respectively, and the postbuckling moments (known as steady-state moments) $M_{+}^{*}$ and $M_{-}^{*}$, for opposite and equal sense bends, respectively. When the peak moments are studied, it is found that opposite sense bends buckle via a snap through mode, whereas equal sense bends buckle via a torsional mode. ${ }^{4}$ This results in higher peak moment magnitudes for opposite sense bends than for equal sense bends.

Three-dimensional folds occur when the fold line is not perpendicular to the lengthwise direction of the tape, as shown earlier in Fig. 1. This causes the tape to fold out of the two-dimensional bending plane. This type of displacement is shown in Fig. 6. However, all three-dimensional folds can be modeled as a combination of bending and twisting moments. To describe fully this motion, a moving coordinate system, equivalent to the two-dimensional case is required.

The moving coordinate system is defined by $x^{\prime}, y^{\prime}$, and $z^{\prime}$, which remains fixed relative to the end of the tape spring as shown in Fig. 7. Any three-dimensional fold can, therefore, be modeled as a combination of rotations about the $y$ axis (bending) and rotations about the $x^{\prime}$ axis (twisting, identically applied to each tape end). The reaction moment and twist about the $x^{\prime}$ axis ( $M$ and $\theta$ for the $y$ axis), are denoted by $T$ and $\gamma$, respectively. (The rotation of $\gamma$ can be seen in Fig. 8.)

Three-dimensional tape spring folds in practical applications are caused by tape springs not being mounted at right angles to the lines 


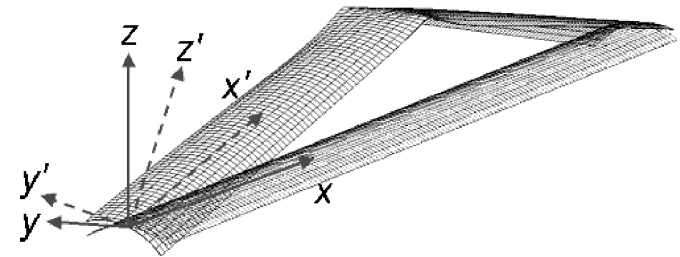

Fig. 7 General coordinate system for three-dimensional tape spring fold.

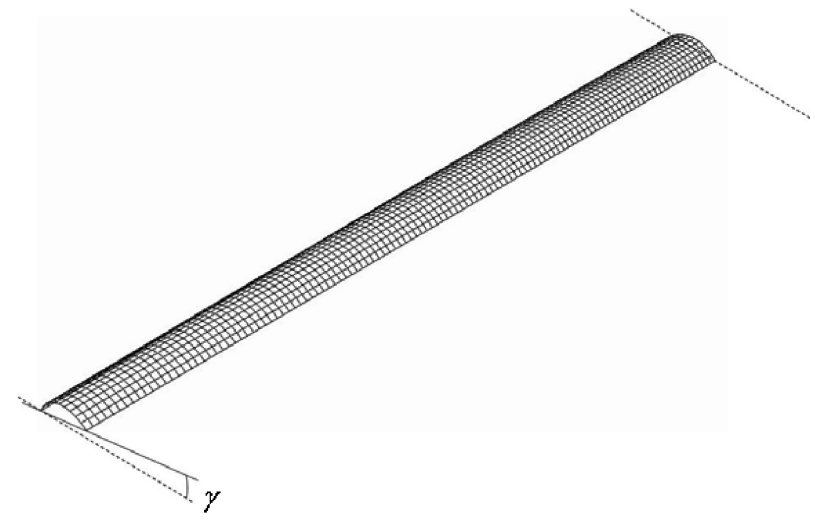

Fig. 8 Tape twisted by $\gamma$.

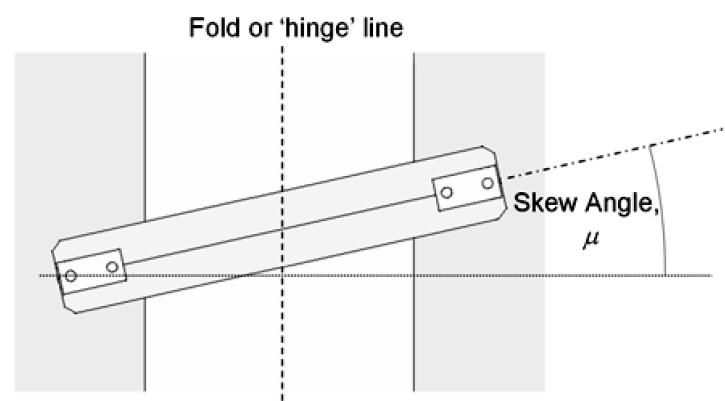

Fig. 9 Plan view of tape spring mounted at skew angle $\mu$.

about which the ends are rotated. The mounting angle is, therefore, defined as the skew angle (Fig. 9).

This skewed mounting creates a slightly different system to the preceding one that lends itself easily to theoretical analyses. In the skewed system, the critical moment is the opening moment about the fold or hinge line, $M_{H}$, as shown in Fig. 9. This is not equivalent to $M$ because this moment acts to push both tape halves away from each other in the theoretical plane ( $x-z$ plane), returning the tape to its straight, unbuckled form (Fig. 10). The theoretical ( $x-z$ plane) plane is, therefore, defined as the surface that contains both tape halves, either side of the central fold. It is called the theoretical plane because it is the plane in which the theoretical surface curvature models can be applied.

These two systems are, therefore, defined as the theoretical system and the skewed system. The theoretical system models a threedimensional fold in one plane as a combination of bending and twisting, whereas the skewed system models the moment about the hinge line, critical for dynamic deployment modeling of a real array. The skewed system is, therefore, the practical application of a three-dimensional tape spring fold.

To investigate the effect of twist $\gamma$ and skew angle $\mu$ on $M$ and $M_{H}$, respectively, an experimental test rig was designed to determine the reaction moments from both two- and three-dimensional tape spring folds. In past research, relatively simple experimental techniques have been used to analyze tape springs, primarily to produce comparative data to prove the accuracy of specific theoretical models. In Ref. 4, the buckling moments were determined by studying the critical lengths at which a horizontally extended tape

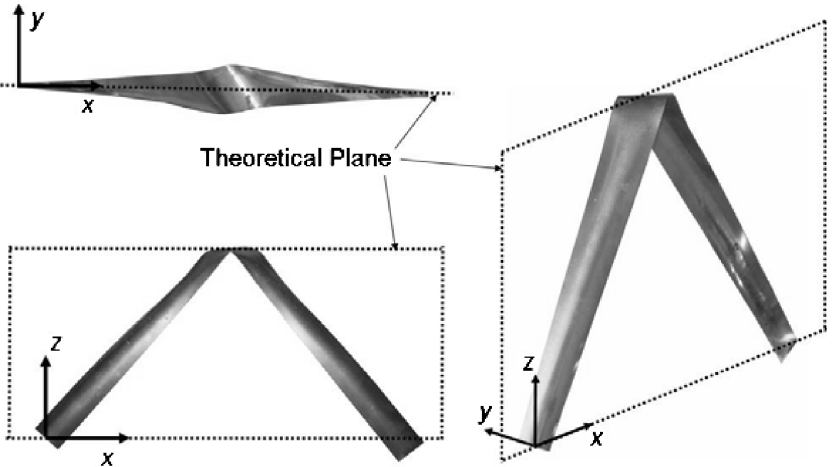

Fig. 10 Theoretical $(x-z)$ plane of tape fold.

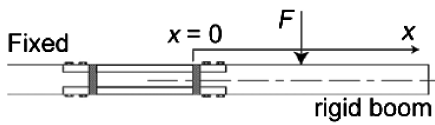

Fig. 11 Experimental model of hinge deflection.
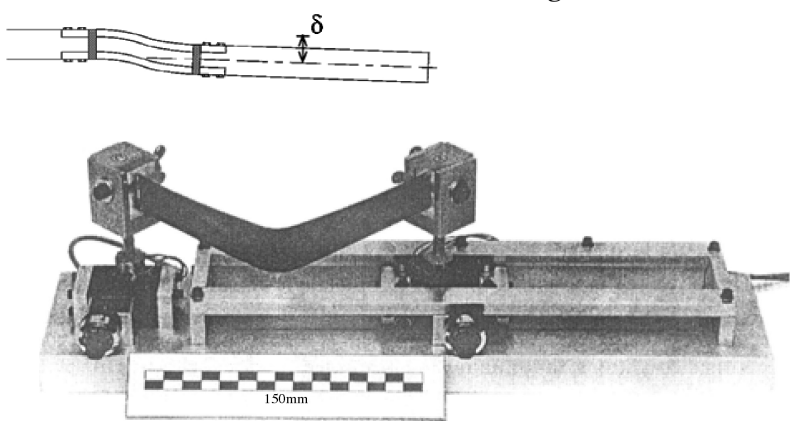

Fig. 12 Test rig used at DSL (courtesy of DSL).

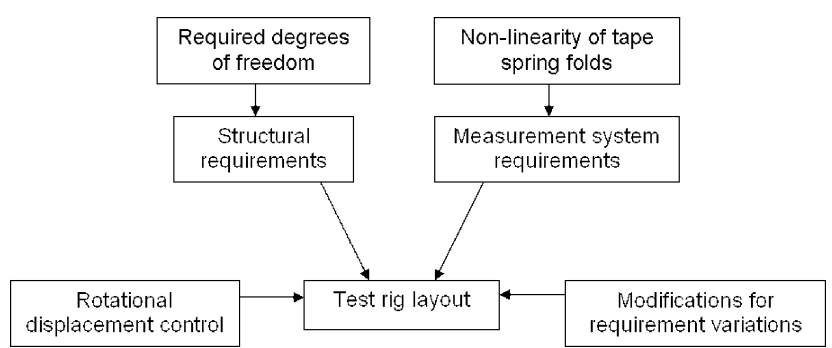

Fig. 13 Test rig design process.

measure collapses under its own weight. More recently, simple experimental models have been used to compare the stiffness of a tape spring hinge. ${ }^{5}$ An example of this is shown in Fig. 11, where a hinge is loaded by a force $F$ to determine its deflection. Similar simple experiments have been performed to validate the stiffness of the three-tape hinge developed by the French company METRAVIB. ${ }^{6}$

More complex rigs have also been used to determine the momentrotation relationships of two-dimensional tape spring folds, the most notable of which is the rig used by the Deployable Structures Laboratory (DSL) in Cambridge, England, United Kingdom. This rig is shown in Fig. 12. The end rotations of this rig are applied manually through two identical gear boxes, and the reaction moments are measured using strain gauges. A full description of this apparatus is given in Ref. 7. However, none of the existing rigs have included the twist degrees of freedom required for a three-dimensional tape spring fold, which significantly increases the complexity of the design.

The final configuration of the experimental test rig developed for this research was the result of a number of design steps, which are outlined in Fig. 13.

Preliminary design decisions were made based on two known factors: the number of degrees of freedom required and the nonlinearity of tape spring folds. These led to the structural requirements 
Fig. 14 Diagram of right tape spring fold. angle three-dimensional

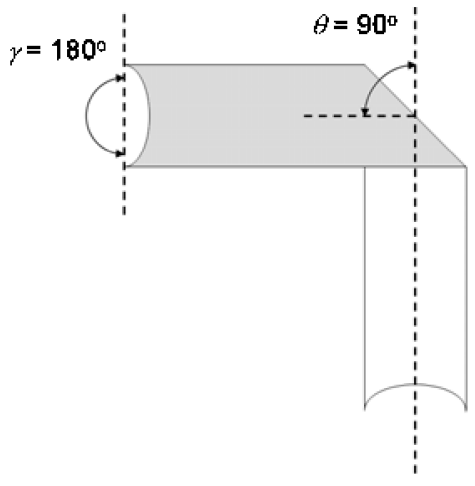

and the measurement system requirements, producing an outline design with similarities to the rig in Fig. 12. However, the inclusion of a twist degree of freedom significantly increased the complexity of the required structure. Both the structural and measurement system requirements then led to the general test rig layout. This layout was closely constrained by the specific requirements of the system. For example, each $y$ (bend) axle rotation had to be greater than $45 \mathrm{deg}$ (to allow the tape to be bent up to an angle greater than $90 \mathrm{deg}$ ), each $x^{\prime}$ (twist) axle had to rotate up to $90 \mathrm{deg}$ (to allow the tape to be twisted up to an angle of $180 \mathrm{deg}$ ), and each axle had to incorporate a mounting for the selected measurement system. These angle requirements would allow the tape samples to simulate a three-dimensional right-angle fold as shown in Fig. 14. Such a fold can be created by mounting a tape spring at a skew angle of $45 \mathrm{deg}$.

The key decision point of the design process was choosing the method of imposing the rotations. The available methods were static locking, where rotations on all four axles are applied manually and locked before the moments are recorded; dynamic loading by hand, where rotations are applied manually and the reaction moments are dynamically recorded; and mechanically applied rotations, where rotations are applied by mechanical means and the reaction moments are dynamically recorded.

The most desirable method of imposing the rotation is by mechanical means because this method allows the angular rate of rotation to be finely controlled and minimizes the shock of the opposite sense bend buckling moments. However, this option was significantly more complex and costly when compared to the other two methods that could be incorporated easily into a rig design. Mechanically applied rotations would have also dramatically added to the problem of friction in the system. The rig has a moving end, which should ideally be a frictionless system. The mass of the moving end was minimized with the use of lightweight materials, and all of the axes of rotation used low friction bearings. The added mass of a mechanically applied rotation system, especially about the twist axis, would have seriously affected the smooth motion of the moving end and the balance of the structure about the $y$ axle. It was, therefore, decided to design the rig to allow both the static locking and dynamic loading methods, the second of which was used in all tests presented in this paper.

The measurement system requirements essentially consisted of low-mass and low-volume restrictions and high accuracy over a potentially wide moment range (from peak moments to low torsional moments). The solution was the strain gauge unit (SGU), shown in Fig. 15.

This simple gauge, essentially a portal frame, consists of three components: load arms, a metal strip, and a strain gauge. As a force is applied across the SGU load arms, the metal strip bends, resulting in an output voltage from the strain gauge that is in proportion to the applied force. To determine the moment about an axle, two SGUs are mounted between a locking lever (rotationally fixed about each axle) and a torque bar (fixed to the freely rotating axle) as shown in Fig. 16.

The measurement of the magnitude of the applied load across the SGU load arms can be optimized by either one or a combination of the following methods: by altering the dimensions of the metal strip

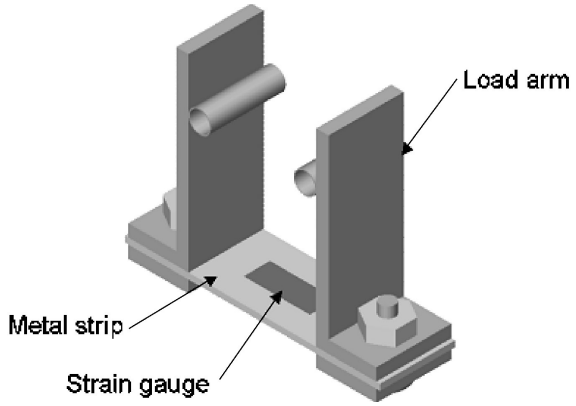

Fig. 15 SGU diagram.

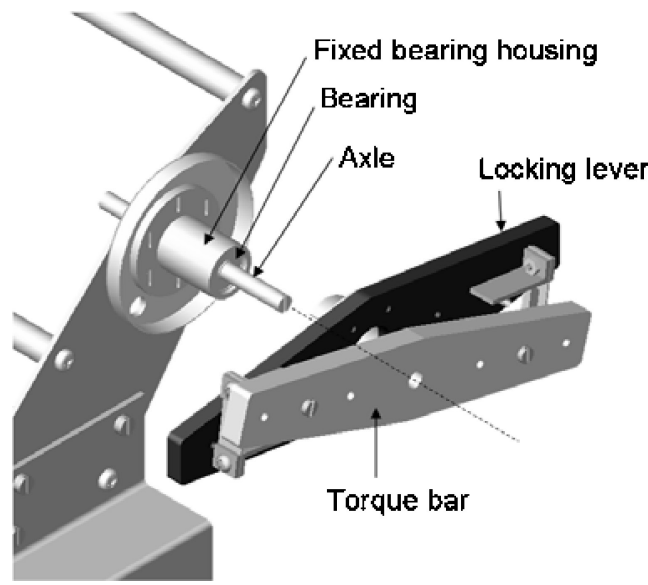

Fig. 16 Measurement system layout.

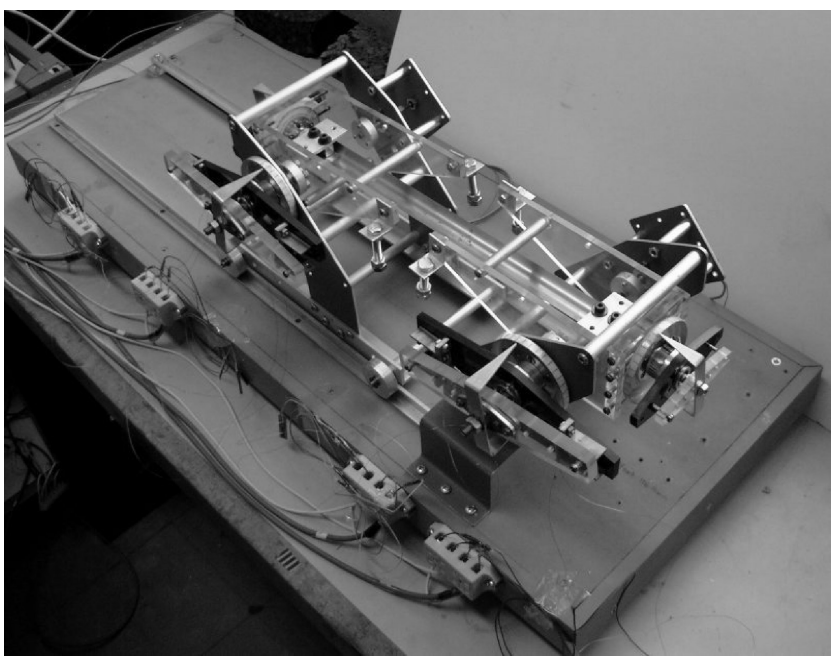

Fig. 17 Configuration for three-dimensional testing in the theoretical plane.

(thickness, width), by altering the radial mounting locations of the SGU, and/or by altering the number of SGUs mounted around an axle. The bend and twist SGUs were optimized for the respective moment magnitudes and were calibrated to be accurate to within $5 \%$.

The structure of the rig was built from aluminum plate mounted on a wooden base board. The structure that rotates about the $y$ axle was designed to be balanced around the axle and was made from perspex to minimize the mass. Extra structural extensions (which can be seen on the far side of the rig in Fig. 17) were constructed to mount potentiometers. These were used as rotation sensors and were calibrated to within $\pm 1 \mathrm{deg}$.

The test rig could be set up to analyze both the theoretical and skewed systems as shown in Figs. 17 and 18, respectively. A more 


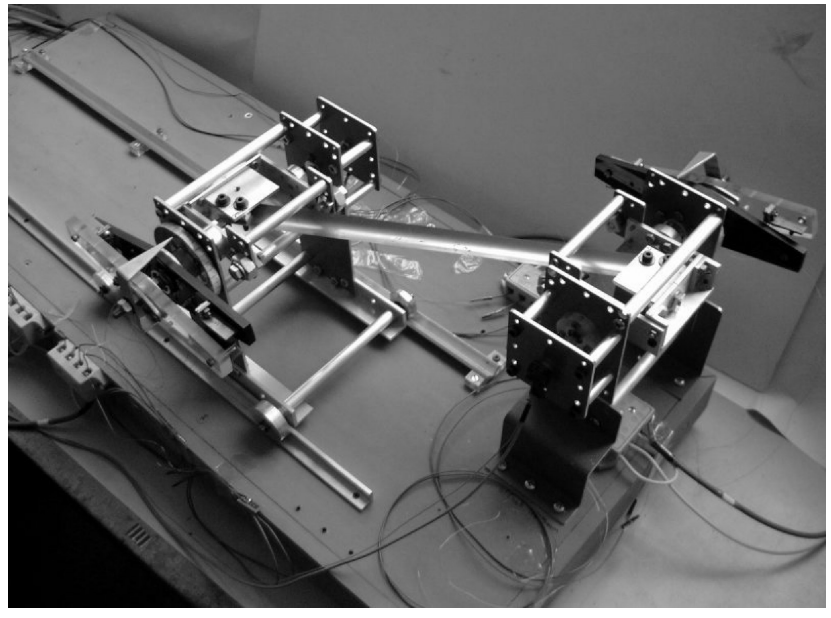

Fig. 18 Configuration for three-dimensional testing on skew-mounted tapes.

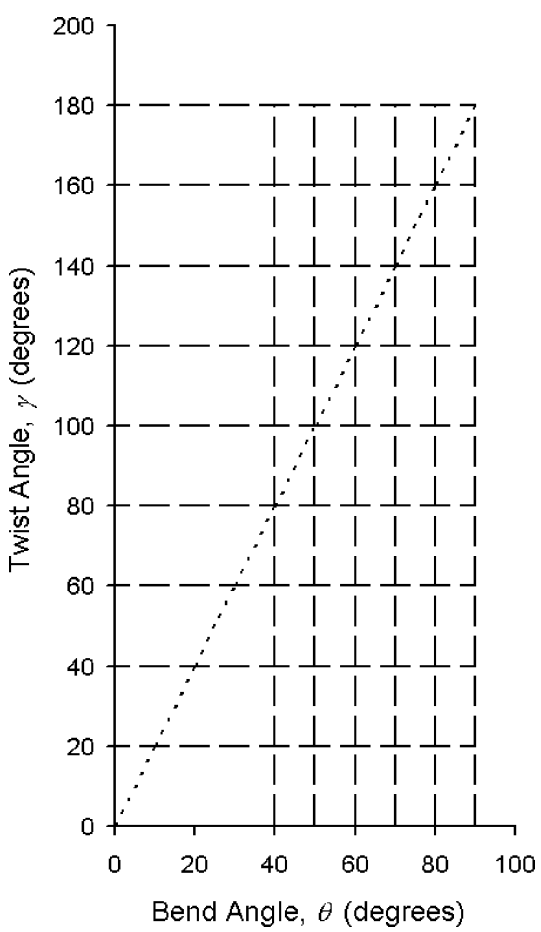

Fig. 19 Data field for experimental tests: ---, test lines and - - - -, perfect fold path for right angle fold.

in-depth description of the test rig and the measurement system may be found in Ref. 8.

Because the dynamic loading method was used to apply the rotations it was impracticable to increase accurately the rotations of four axes simultaneously. Any manually applied rotational input into the $x^{\prime}$ axle distorts the sensor output from the $y$ axle. The threedimensional system under investigation essentially produces a data field of $M$ and $T$ defined by two variable parameters, $\theta$ and $\gamma$, as shown in Fig. 19. Therefore, to analyze this system within the physical limits imposed by the test rig, one parameter can be held constant while the other is used as a variable. This method implies that only two rotations need to be applied for any single test

Figure 19 shows a summary of all of the tests performed along with a line denoting a perfect fold path for a right-angle threedimensional fold. For this study, a perfect fold path is defined as being a path that has a constant mathematical relationship between the angles $\theta$ and $\gamma$. In Fig. 19, the path is defined by $\gamma=2 \theta$. No testing was performed at constant bend angles of less than $40 \mathrm{deg}$ because of the instability of the fold at such low angles.
The accuracy of the test rig was a primary concern throughout the design process, which led to the material choices to minimize mass and component choices to minimize the static friction of the rotation axles and the resistance of the moving end. It was found that the static friction torque was negligible even for the optimized sensors designed to measure the tape moments. The friction of the moving end along the track was also very low. Tests were performed using a stiff steel bar to determine the compliance of the rig, and it was found that a peak moment of $600 \mathrm{~N} \cdot \mathrm{mm}$ resulted in a measured rotation of $2.5 \mathrm{deg}$. Therefore, for every $100 \mathrm{~N} \cdot \mathrm{mm}$ of applied moment, the rig deflects giving an angle reading of $0.42 \mathrm{deg}$. It was found from these tests that these magnitudes would only be significant for opposite sense bend peak moments. Initial tests showed that the test rig produced accurate data; however, inaccuracies were produced primary due to the complexities of the system being studied. For example, it was found from pretests on samples of carpenter tape that after repeated buckling the peak moment magnitudes would slightly reduce. This is caused by the development of imperfections in the tape at the location of buckling. Complications are also present when attempting to determine accurately the steadystate moment. Analytical models define the postbuckled moment to be proportional to the cube of the tape thickness. ${ }^{9}$ The experimental thickness range of the specimens (from the same original roll) was found to be $0.10668-0.12250 \mathrm{~mm}$, creating a possible moment variation of 14 and $25 \mathrm{~N} \cdot \mathrm{mm}$ for opposite and equal sense bends, respectively. From extensive experimental testing (40 and 50 test runs for opposite and equal sense bends, respectively), it was found that more than $90 \%$ of the test runs produced moment magnitudes that remained consistently within these steady-state moment ranges. The test runs that went outside these ranges did so briefly due to oscillatory movement caused by the motion of the moving end. In contrast, the torsional moments were very stable (due to the stationary nature of the fold and the moving end). Because tape spring moments are, therefore, very variable, this work aims to identify trends that were found to be repeated over a number of different tape samples. The maximum stress in the samples throughout the test campaign was kept less than $\sigma_{\text {yield }}$.

\section{Two-Dimensional Tests}

Comparative two-dimensional fold data were produced by experimentally investigating six tape spring samples of various lengths. The samples, all originating from the same tape spring roll, were bent to form two-dimensional tape spring folds with bend angles of $\theta= \pm 90 \mathrm{deg}$. It was necessary to perform the two-dimensional tests first to determine accurately the true peak moment magnitude before the samples developed imperfections. The properties of the samples can be seen in Table 1, and the moment data for the opposite and equal sense bends analyzed are shown in Tables 2 and 3, respectively. Three repeats were performed for each test configuration (denoted by T1, T2, and T3).

The tape spring properties were determined from various experimental tests and have been used in comparative theoretical analyses to be presented in future publications.

From the opposite sense bend data, it can be seen that the peak moment results are generally scattered around a magnitude of $600 \mathrm{~N} \cdot \mathrm{mm}$. The peak moment magnitude only increases for very short tape lengths. This trend has also been found in past work ${ }^{3}$ and is primarily due to end effects. For longer tape lengths, it can be concluded that there is no significant variation in the opposite sense peak moment as the length of the tape increases. From the equal

Table 1 Tape spring properties

\begin{tabular}{lc}
\hline \hline Property & Value \\
\hline$E$ & $195.3 \times 10^{3} \mathrm{~N} \cdot \mathrm{mm}^{2}$ \\
$R_{y}$ & $15.37 \mathrm{~mm}$ \\
$\alpha$ & $1.719 \mathrm{rad}$ \\
$\kappa_{y, 0}$ & $0.065 \mathrm{~mm}^{-1}$ \\
$t$ & $0.10668-0.12250 \mathrm{~mm}$ \\
$\sigma_{\text {yield }}$ & $1190 \mathrm{MPa}$ \\
\hline \hline
\end{tabular}


Table 2 Experimental moments for opposite sense bends

\begin{tabular}{|c|c|c|c|c|c|c|}
\hline \multirow{2}{*}{$\begin{array}{l}\text { Tape } \\
\text { sample }\end{array}$} & \multirow[b]{2}{*}{$L, \mathrm{~mm}$} & \multicolumn{3}{|c|}{ Peak moment, $\mathrm{N} \cdot \mathrm{mm}$} & \multirow{2}{*}{$\begin{array}{c}\text { Average peak } \\
\text { moment, } \mathrm{N} \cdot \mathrm{mm}\end{array}$} & \multirow{2}{*}{$\begin{array}{l}\text { Average steady-state } \\
\text { moment, } \mathrm{N} \cdot \mathrm{mm}\end{array}$} \\
\hline & & $\mathrm{T} 1$ & $\mathrm{~T} 2$ & $\mathrm{~T} 3$ & & \\
\hline 1 & 98 & 756 & 746 & 741 & 748 & 61 \\
\hline 2 & 177 & 626 & 637 & 624 & 629 & 43 \\
\hline 3 & 267 & 592 & 592 & 595 & 593 & 48 \\
\hline 4 & 267 & 593 & 604 & 607 & 602 & 56 \\
\hline 5 & 433 & 578 & 577 & 595 & 584 & 42 \\
\hline 6 & 433 & 618 & 600 & 602 & 607 & 53 \\
\hline
\end{tabular}

Table 3 Experimental moments for equal sense bends

\begin{tabular}{|c|c|c|c|c|c|c|}
\hline \multirow{2}{*}{$\begin{array}{l}\text { Tape } \\
\text { sample }\end{array}$} & \multirow[b]{2}{*}{$L, \mathrm{~mm}$} & \multicolumn{3}{|c|}{ Peak moment, $\mathrm{N} \cdot \mathrm{mm}$} & \multirow{2}{*}{$\begin{array}{c}\text { Average peak } \\
\text { moment, } \mathrm{N} \cdot \mathrm{mm}\end{array}$} & \multirow{2}{*}{$\begin{array}{c}\text { Average steady-state } \\
\text { moment, } \mathrm{N} \cdot \mathrm{mm}\end{array}$} \\
\hline & & $\mathrm{T} 1$ & $\mathrm{~T} 2$ & $\mathrm{~T} 3$ & & \\
\hline 1 & 98 & -178 & -179 & -176 & -178 & -33 \\
\hline 2 & 177 & -108 & -110 & -107 & -108 & -31 \\
\hline 3 & 267 & -102 & -102 & -102 & -102 & -38 \\
\hline 4 & 267 & -95 & -98 & -95 & -96 & -33 \\
\hline 5 & 433 & -89 & -87 & -87 & -88 & -29 \\
\hline 6 & 433 & -91 & -91 & -93 & -92 & -31 \\
\hline
\end{tabular}

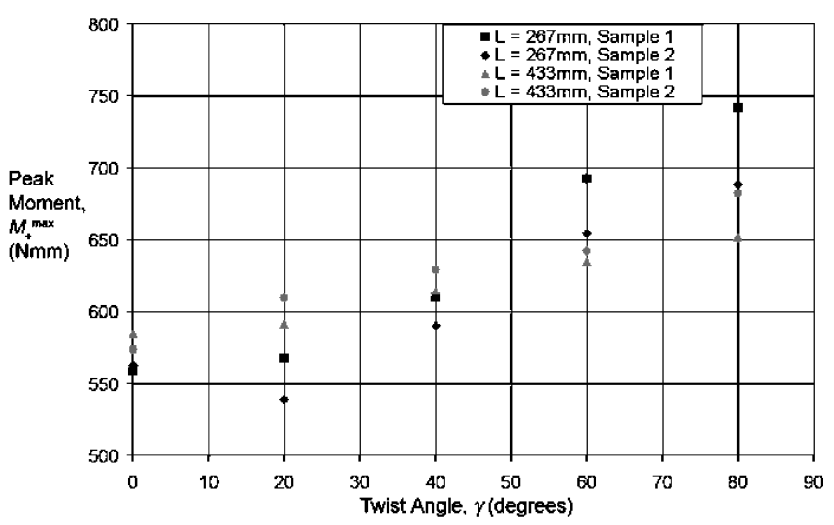

Fig. 20 Opposite sense bend peak moment increase.

sense bend peak moment data, it can be concluded that there is a general trend of reducing peak moment as the tape length increases. However, for both bend cases, there seems to be no correlation between tape length and the steady-state moment (other than end effects). From previous work, ${ }^{10}$ it is known that increasing length causes the peak moment opposite sense bend angle $\theta_{+}^{\max }$ to increase. This phenomenon was repeated in these tests resulting in a peak angle range of 8-18 deg for opposite sense bends. Here $\theta_{-}^{\max }$ was found to range between 7 and $14 \mathrm{deg}$.

\section{Peak Moment Analysis}

After the establishment of the general moment trends for a twodimensional tape spring fold, the experimental test rig was used to investigate the effect of $\gamma$ and $\mu$ on the peak moment magnitudes. This involved experimental analyses of both the theoretical and skewed systems and was performed repeatedly by keeping $\gamma$ and $\mu$ constant while $\theta$ was changed.

To study the influence of $\gamma$ on $M_{+}^{\max }$ and $M_{-}^{\max }$, four tape spring samples of two different lengths, $L=267$ and $433 \mathrm{~mm}$, were subjected to experimental tests. The opposite sense bend peak moment results are shown in Fig. 20. It can be seen from Fig. 20 that the initial data at $\gamma=0 \mathrm{deg}$ correspond to the two-dimensional analysis data in Table 2, although the magnitudes are slightly lower due to the developed imperfections in the samples. However, as a twist is applied, the peak moment $M_{+}^{\max }$ rises. This result was consistent for all tests.

To protect the rig and the sensors from damage (which were already operating at their sensitivity limit), the tests were limited to five twist angles up to $80 \mathrm{deg}$.

It was discovered from these tests that an imposed twist angle, when constrained by end clamps, caused a bend in the tape in the

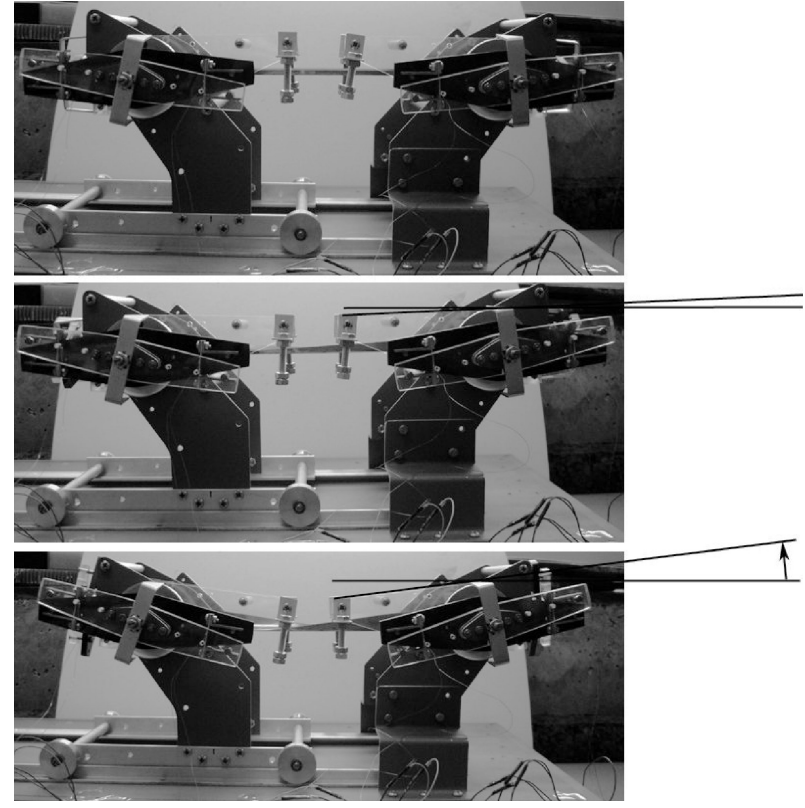

Fig. 21 End depression and tape bend caused by three different initial twist angles.

equal sense direction. This coupling of the bend and twist rotations is a kinematic effect and can be seen clearly in Fig. 21 as the depression angle of each end increases with twist angle. The larger the initial twist, the higher the initial bend angle is and the higher the transverse curvature in the center of the tape. Applied rotations in the opposite sense bend direction, after the initial twist has been applied, results in a direct strain being applied into the tape to make $\theta>0$ deg before buckling can occur. Therefore, $M_{+}^{\max }$ increases as the initial twist angle increases. Here $\theta_{+}^{\max }$ was also found to reduce as $\gamma$ increased, resulting in peak moment bend angles of less than $8 \mathrm{deg}$.

The relationship between the initial twist angle and the initial bend angle also modifies $M_{-}^{\max }$. The impact of this initial twist is clearly shown in the experimental data shown in Fig. 22. The rise in $\gamma$ causes the magnitude of $\theta_{-}^{\max }$ to increase, and as equal sense bends buckle in a torsional mode, the presence of twist aids the formation of a localized fold resulting in a reduction in the peak moment. Figure 19 shows this trend, which was repeated for all four of the samples tested.

The data predict average peak moment reductions of 16 and $37 \%$ for tape lengths of 433 and $267 \mathrm{~mm}$, respectively. Therefore, it can 
Table 4 Experimental peak moments for skew-mounted opposite sense bends

\begin{tabular}{lccccc}
\hline \hline $\begin{array}{l}\text { Length } \\
L, \mathrm{~mm}\end{array}$ & $\begin{array}{c}\text { Skew angle } \\
\mu, \mathrm{deg}\end{array}$ & \multicolumn{2}{c}{ Peak moment, N $\cdot \mathrm{mm}$} & \begin{tabular}{c} 
Average peak \\
\cline { 3 - 4 } moment, N $\cdot \mathrm{mm}$
\end{tabular} \\
\hline 98 & 0 & $\mathrm{~T} 1$ & $\mathrm{~T} 2$ & $\mathrm{~T} 3$ & 748 \\
98 & 12.6 & 744 & 750 & 749 & 748 \\
267 & 0 & 592 & 592 & 595 & 593 \\
267 & 0 & 593 & 604 & 607 & 602 \\
267 & 29.3 & 515 & 510 & 522 & 516 \\
267 & 29.3 & 534 & 523 & 533 & 530 \\
433 & 0 & 578 & 577 & 595 & 584 \\
433 & 0 & 618 & 600 & 602 & 607 \\
433 & 18.2 & 563 & 556 & 551 & 557 \\
433 & 18.2 & 574 & 572 & 573 & 573 \\
567 & 14.0 & 557 & 549 & 557 & 554 \\
567 & 14.0 & 583 & 561 & 560 & 568 \\
\hline \hline
\end{tabular}

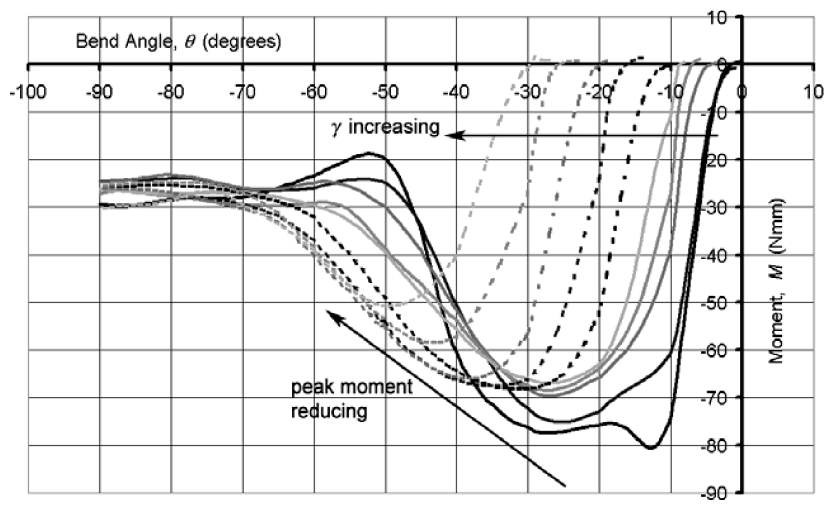

Fig. 22 Effect of twist on equal sense bend moment, $L=267 \mathrm{~mm}$, sample 1.

be seen that the impact of $\gamma$ on $M_{+}^{\max }$ and $M_{-}^{\max }$ is much more significant for the short-tape sample than for the long tape. This is due to the higher rotation of the principal curvature lines at the fold region for the shorter tape. ${ }^{11}$ Therefore, as $L$ increases, the effect of $\gamma$ on $M_{+}^{\max }$ and $M_{-}^{\max }$ reduces.

To investigate the effect of $\mu$ on the peak hinge moment, testing was performed on six tape samples of three different lengths, $L=267,433$, and $567 \mathrm{~mm}$, resulting in the experimental analysis of three different skew angles. A summary of the opposite sense peak moment results is shown in Table 4.

It was concluded earlier from the data in Table 2 that there was no apparent relationship between the tape length and the peak moment outside the influence of end effects. However, the data in Table 4 suggest that the opposite sense peak moment is affected by the skew angle. In these test cases, the application of the skew angle changes the location of the buckling point of on the tape, minimizing the effect of the imperfection development. When the data for the samples of length $L=267$ and $433 \mathrm{~mm}$ are compared, it can be seen that as the skew angle increases the peak moment reduces. This is probably caused by the reduction in curvature along the fold line as $\mu$ increases. $\Lambda_{0+}^{\max }$ also was found to increase as the tape length increased, resulting in a peak bend angle range of 16-25 deg for the data in Table 4.

The equivalent equal sense bend results are displayed in Table 5. From these data, it could be argued that an increase in the skew angle causes a slight peak moment reduction. However, the magnitude changes are not large enough to provide a definite verification of this conjecture. The peak moment reduction, as a result of increasing tape length, is a much more dominant data trend. $\Lambda_{0-}^{\max }$ was found to occur at around $10 \mathrm{deg}$ for the tests shown in Table 5. However, for the longer tape samples, this angle corresponds to the formulation of two localized folds that converged to a single centralized fold at a higher bend angle.
Table 5 Experimental peak moments for skew mounted equal sense bends

\begin{tabular}{lccccc}
\hline \hline \multirow{2}{*}{$\begin{array}{l}\text { Length } \\
L, \mathrm{~mm}\end{array}$} & $\begin{array}{c}\text { Skew angle } \\
\mu, \text { deg }\end{array}$ & \multicolumn{2}{c}{ Peak moment, $\mathrm{N} \cdot \mathrm{mm}$} & \multirow{2}{*}{$\begin{array}{c}\text { Average peak } \\
\text { moment, N } \cdot \mathrm{mm}\end{array}$} \\
\hline 98 & 0 & -178 & -179 & -176 & -178 \\
98 & 12.6 & -145 & -147 & -147 & -146 \\
267 & 0 & -102 & -102 & -102 & -102 \\
267 & 0 & -95 & -98 & -95 & -96 \\
267 & 29.3 & -88 & -90 & -88 & -89 \\
267 & 29.3 & -98 & -96 & -94 & -96 \\
433 & 0 & -89 & -87 & -87 & -88 \\
433 & 0 & -91 & -91 & -93 & -92 \\
433 & 18.2 & -86 & -87 & -87 & -86 \\
433 & 18.2 & -86 & -87 & -88 & -87 \\
567 & 14.0 & -80 & -81 & -78 & -79 \\
567 & 14.0 & -76 & -75 & -74 & -75 \\
\hline \hline
\end{tabular}

Table 6 Opposite sense bend steady-state moment

\begin{tabular}{lc}
\hline \hline $\begin{array}{l}\text { Twist angle } \\
\gamma, \text { deg }\end{array}$ & $\begin{array}{c}\text { Average steady-state moment } \\
M_{+}^{*}, \mathrm{~N} \cdot \mathrm{mm}\end{array}$ \\
\hline 0 & 39 \\
20 & 38 \\
40 & 40 \\
60 & 45 \\
80 & 44 \\
\hline
\end{tabular}

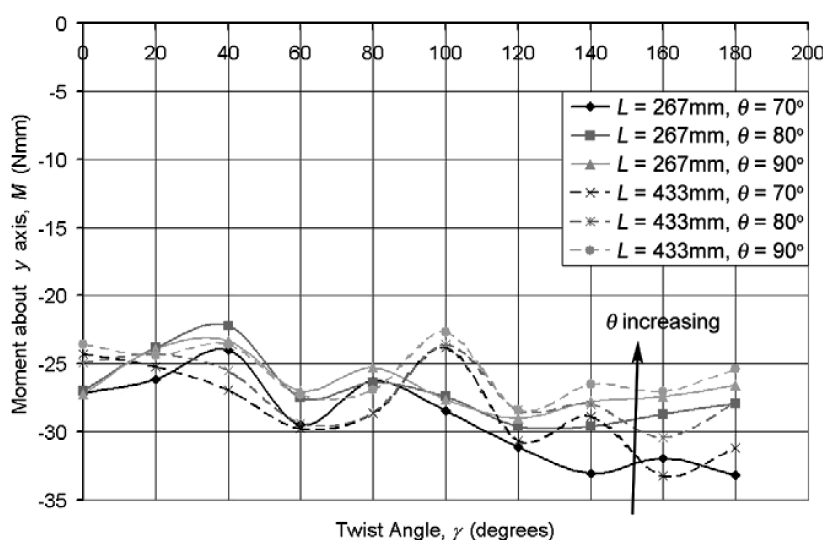

Fig. 23 Equal sense bend steady-state moment for increasing values of $\gamma$.

\section{Steady-State Moment Analysis}

After the determination of the influence of twist and skew angle on the peak buckling moments of a tape spring fold, it was necessary to perform an equivalent analysis on the postbuckling moments. When it is observed that surface area deployments on satellites consist mostly of rotations greater than $90 \mathrm{deg}$ and usually $180 \mathrm{deg}$, then it can be concluded that the vast majority of any tape spring deployment consists of tape springs unfolding in the buckled state. Therefore, any influence of $\gamma$ and $\mu$ on the opening moment is of particular importance. These tests were again performed repeatedly by keeping $\gamma$ and $\mu$ constant while $\theta$ was changed.

The average result from the opposite sense bend tests on samples of length $L=267 \mathrm{~mm}$ is shown in Table 6. It can be seen that as $\gamma$ increases the average opposite sense steady-state moment increases. However, the average increase of $M$ over this twist range is only around $13 \%$. The results of these tests are, therefore, inconclusive because the potential moment variation due to the thickness tolerance exceeds this value. More significant variations in $M_{+}^{*}$ could be achieved if the samplers were twisted to $\gamma=180 \mathrm{deg}$. However, this would probably cause permanent damage to the tape sample.

The equal sense bend data analyzing $M$ are shown in Fig. 23. Because of the complex interaction between $M$ and $\gamma$ (shown earlier in 
Fig. 22), it was necessary to analyze the full moment rotation relationship as $\gamma$ increased. This study was performed on four tape samples of two lengths. It can be seen from Fig. 22 that, as $\gamma$ increases, the transition of $M$ between the peak moment and the steady-state moment becomes smoother. This is due to the presence of twist because high values of $\gamma$ significantly aid the formation of a localized fold. The angle at which the localized fold forms, hence, the steadystate moment begins, is, therefore, highly variable and dependent on $\gamma$. To investigate the affect of twist on the steady-state moment, $M$ is plotted at bend angles of $-70,-80$, and -90 deg as shown in Fig. 23. In Fig. 23, the data points are displayed with interpolated curves to improve the clarity of the diagram.

In Fig. 23, each twist angle (denoted by the vertical lines) displays the results of two tests, one for $L=267 \mathrm{~mm}$ and one for $L=433 \mathrm{~mm}$. Because of the moment about the $y$ axis being proportional to the cube of the tape thickness, ${ }^{9}$ fluctuations in the steady-state moment are seen over the range of twist angles. However, the vertical separation of these points increases noticeably when the twist angle increases past $100 \mathrm{deg}$. This is caused by the changeable transition of $M$ between peak and steady state that only noticeably affects the higher bend angles at high twist angles. These data conclude that, as the bend angle increases, the impact of $\gamma$ on $M$ reduces. This can result in no increase in the opening moment for a bend angle of $-90 \mathrm{deg}$. Because the rise in $M_{-}^{*}$ is due to the opening moment transition, the value of $\gamma$ at $M_{-}^{\max }$ and the subsequent change in $\gamma$ defines the angle over which the moment transition takes place. However, for large bend angles, where $\theta$ is around $90 \mathrm{deg}$, it can be assumed that $\gamma$ has an insignificant affect on $M$.

To determine the experimental data trends for skew-mounted tape springs, the postbuckling moment data were overlaid on the twodimensional experimental results. These data are shown in Figs. 24 and 25 . Two tape lengths ( $L=267$ and $433 \mathrm{~mm}$ ) were used in this analysis.

Although it is known that the tape thickness tolerance causes a scatter variation in the steady-state moment, the trends of the averages in Figs. 24 and 25 are representative of all of the 10 repeat tests performed for each configuration. The repeat tests also remain within the predicted moment tolerance of $\pm 7 \mathrm{~N} \cdot \mathrm{mm}$ and $\pm 13 \mathrm{~N} \cdot \mathrm{mm}$ for opposite and equal sense bends, respectively.

Standard analytical models based on shell theory and finite element simulations tend to predict that in general an increase in the skew angle results in a reduction in the hinge moment. ${ }^{9}$ This trend is very clearly represented in Fig. 25 for a tape length of $L=267 \mathrm{~mm}$. In this case, a skew angle of $30 \mathrm{deg}$ produces a notable reduction in $M_{H}$. However, when the skew angle decreases for the longer tape length, there is no magnitude change in the hinge moment. Similar trends can be seen from the opposite sense bend data for bend angles of less than $40 \mathrm{deg}$. However, for higher bend angles, the skew-mounted tape springs produce a higher hinge moment. This is due to the higher torsion moments encountered for opposite sense bends at large bend angles. These torsional moments can, therefore, have a noticeable effect on $M_{H}$ when their magnitude is significant.

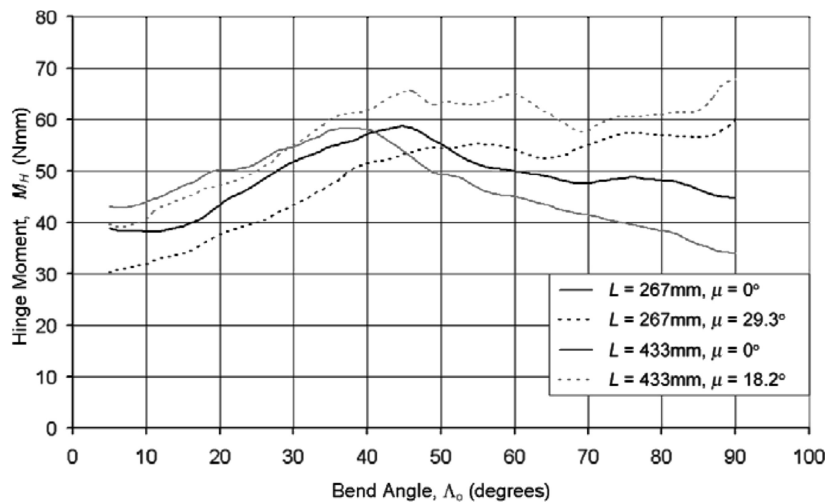

Fig. 24 Average skewed system data overlaid on two-dimensional bend data for opposite sense bend.

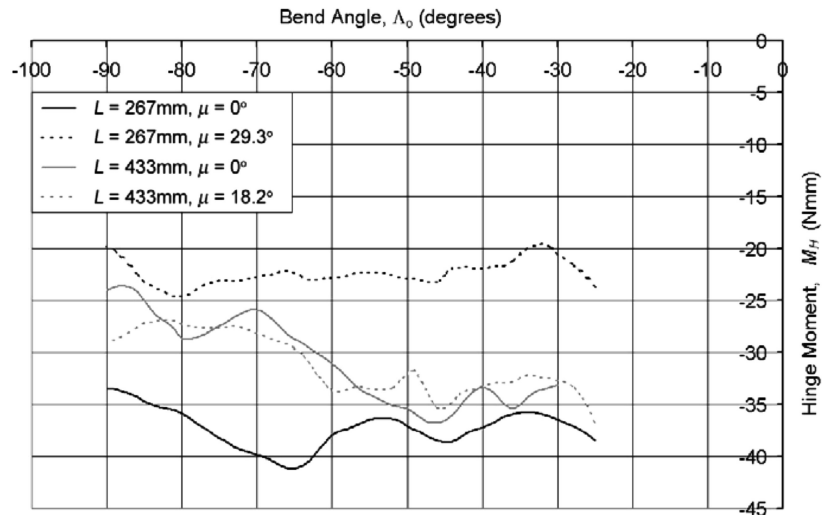

Fig. 25 Average skewed system data overlaid on two-dimensional bend data for equal sense bend.

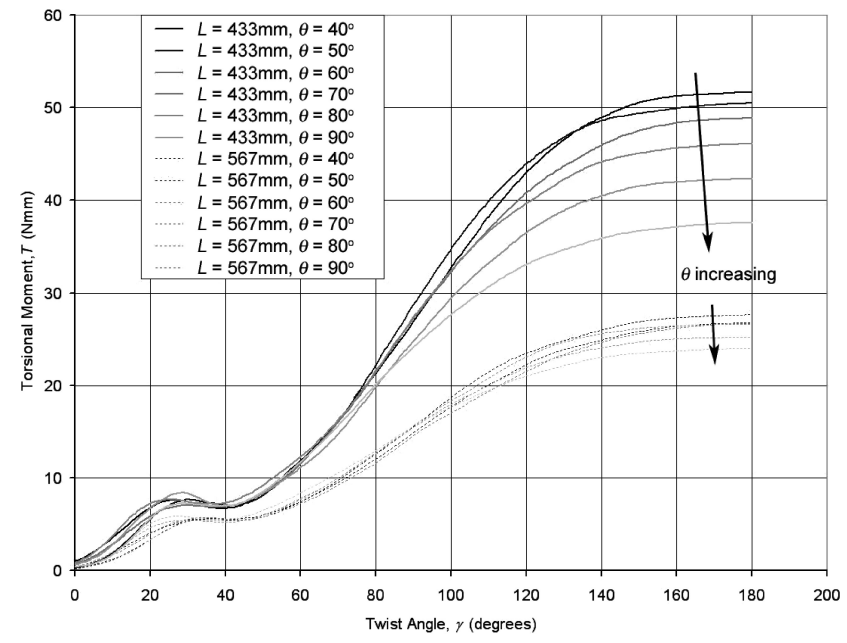

Fig. 26 Opposite sense bend data investigating $T$ for increasing values of $\theta$.

\section{Torsional Moment Analysis}

To investigate the influence of tape length and bend angle on the torsional moment, the test rig was set up to analyze tape spring folds in the theoretical system. The opposite sense bend twist analysis was performed on four tape samples of two different lengths because it was found that the torsional moments produced from tape lengths shorter than $L=400 \mathrm{~mm}$ resulted in moment magnitudes beyond the measurement range of the sensors. The corresponding equal sense bend twist analysis was performed on five tape samples of three different lengths ( $L=267,433$, and $567 \mathrm{~mm})$. The data are shown in Figs. 26 and 27 for opposite and equal sense bends, respectively.

The torsional moment data were found to be very stable when compared to the steady-state moment variation. This was due to the stationary moving end and fold point, as $\theta$ was held constant while $\gamma$ changed.

It can be seen from Fig. 26 that there is consistently an initial peak in the torsional moment. From previous investigations, this was found to be due to a jump (or settling) of the principal curvature lines at the fold point. When the magnitude of the results are compared, it is clear that the peak value of $T$ reduces as $L$ increases. This trend is consistent for both bend directions. It can also be concluded from the data that, for opposite sense bends, as $\theta$ increases, the maximum value of $T$ reduces.

This trend is reversed for equal sense bends, as shown in Fig. 27. The impact of $\theta$ tends to become more significant as the twist angle increases and the tape length reduces. This is again due to the higher principal curvature rotation of shorter tape lengths, a property noticed earlier in the analysis of the peak moments. 


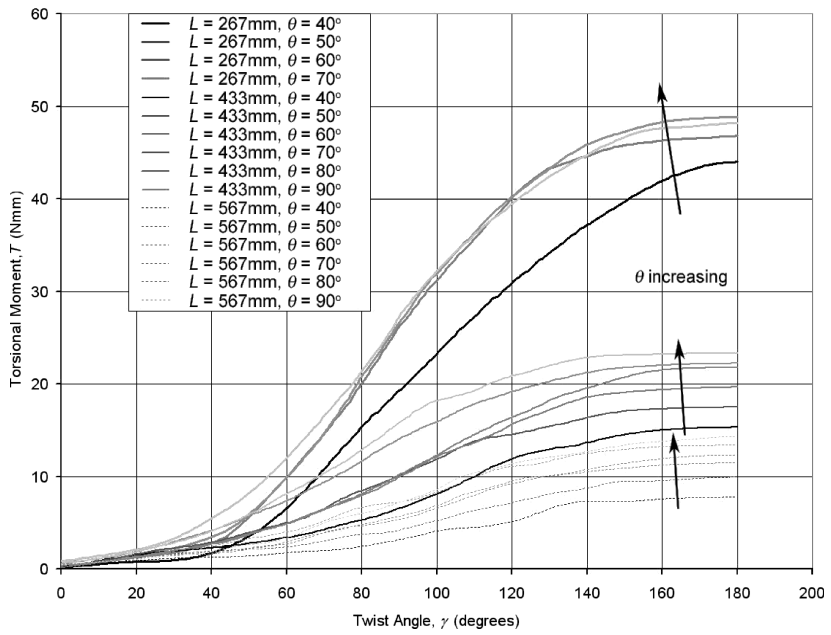

Fig. 27 Equal sense bend data investigating $T$ for increasing values of $\theta$.

\section{Conclusions}

The aim of this work was to investigate experimentally the properties of three-dimensional tape spring folds and to identify general data trends as the rotation parameters were changed. Equivalent theoretical models have been developed alongside this experimental work and will be presented in a future publication. The experimental work presented analyzed tape springs in both the theoretical and skewed systems to investigate the effect of twist angle $\gamma$ on the peak moment $M^{\text {max }}$, skew angle $\mu$ on the peak hinge moment $M_{H}^{\text {max }}$, twist angle $\gamma$ on the steady-state moment $M^{*}$, skew angle $\mu$ on the steady-state hinge moment $M_{H}^{*}$, and bend angle $\theta$ on the torsional moment $T$. The general conclusions of these analyses are as follows:

1) $M_{+}^{\max }$ increases as the twist angle increases.

2) $M_{-}^{\max }$ decreases as the twist angle increases.

3) Both $M_{H+}^{\max }$ and $M_{H-}^{\max }$ decrease with increasing skew angle.

4) An increase in the twist angle smoothes the transition between $M_{-}^{\max }$ and $M_{-}^{*}$, which may increase $M_{-}^{*}$ for certain bend angles.

5) An increase in the skew angle causes $M_{H}^{*}$ to reduce until the magnitude of $T$ becomes significant.

6) $T_{+}$decreases as the bend angle increases.

7) $T_{-}$increases as the bend angle increases.
The tests were undertaken with the final aim of understanding the opening hinge moment of a three-dimensional tape spring fold mounted at any skew angle to the fold line. It was determined from these tests that as the skew angle rose above a value of $\mu=20 \mathrm{deg}$ the hinge moment reduced about the fold line. This hinge moment reduction occurs until the higher torque magnitudes in the theoretical plane begin to dominate $M_{H}$, causing it to rise above the magnitude of $M$. This most noticeably appears in the opposite sense bend data, due to the higher torque magnitude of opposite sense bends. It can, therefore, be concluded that for low skew angles $M_{H}=M$.

\section{References}

${ }^{1}$ Paine, M. D., and Gabriel, S. B., "A Micro-Fabricated Colloidal Thruster Array," AIAA Paper 2001-3329, July 2001.

${ }^{2}$ Gray, A., Modern Differential Geometry of Curves and Surfaces, CRC Press, Boca Raton, FL, 1993, pp. 270, 271.

${ }^{3}$ Seffen, K., and Pellegrino, S., "Deployment Dynamics of Tape Springs," Proceedings of the Royal Society of London, Series A: Mathematical and Physical Sciences, Vol. A455, No. 1983, 1999, pp. 1003-1048.

${ }^{4}$ Mansfield, E., "Large-Deflection Torsion and Flexure of Initially Curved Strips," Proceedings of the Royal Society of London, Series A: Mathematical and Physical Sciences, Vol. A334, No. 1598, 1973, pp. 279-298.

${ }^{5}$ Oya, K., and Onoda, J., "Characteristics of Carpenter Tape Hinge Made of TiNi Alloy," AIAA Paper 2002-1358, April 2002

${ }^{6}$ Givois, D., Sicre, J., and Mazoyer, T., "A Low Cost Hinge for Appendices Deployment: Design, Test and Applications," Proceedings of the 9th European Space Mechanisms and Tribology Symposium [CD-ROM], ESA, Liège, Belgium, 2001.

${ }^{7}$ Fischer, A., "Bending Instabilities of Thin-Walled Transversely Curved Metallic Springs," Dept. of Engineering, Cambridge Univ., Rept. CUED/ D-STRUCT/TR 154, Cambridge, England, U.K., 1995.

${ }^{8}$ Walker, S., and Aglietti, G., "Experimental Testing of Tape Springs Folded in Three Dimensions," 53rd International Astronautical Congress, Paper IAC-02-I.1.07, 2002.

${ }^{9}$ Walker, S., "A Study of Three Dimensional Tape Spring Folds for Space Applications," Ph.D. Dissertation, School of Engineering Sciences, Univ. of Southampton, Southampton, England, U.K., Dec. 2004.

${ }^{10}$ Seffen, K., "Deployment Dynamics of Tape Springs," Ph.D. Dissertation, Dept. of Engineering, Cambridge Univ., Cambridge, England, U.K., 1997.

${ }^{11}$ Walker, S. J. I., and Aglietti, G., "Study of the Dynamics of ThreeDimensional Tape Spring Folds," AIAA Journal, Vol. 42, No. 4, 2004, pp. $850-856$.

B. Sankar Associate Editor 